\title{
Environmental and Forensic Applications of Field-Portable GC-MS: An Overview
}

\author{
B. A. Eckenrode \\ Forensic Research Unit, Federal Bureau of Investigation, Quantico, Virginia, USA
}

\begin{abstract}
GC-MS can provide analytical information that is most reliable for many types of organic analyses. As field-portable GC-MS analytical systems evolve, the application scenarios have diversified as well. With the development of rugged fieldable systems, these instruments were demonstrated to be usable in the harsh environment of the jungle and in chemical demilitarization or military reconnaissance situations. Continuous unattended operations of a GC-MS for 12- or 24-hour monitoring applications in the field have been shown to be possible. A real-time algorithm strategy is proposed, which can be developed to aid in the advancement of field-portable mass spectrometry applied to chemical warfare agent analysis in military vehicles and can be used to raise the standard for field data quality. Each of these capabilities is discussed with the intent on reviewing analysis situations that can be expanded because of developments in field GC-MS instrumentation. (J Am Soc Mass Spectrom 2001, 12, 683-693) (c) 2001 American Society for Mass Spectrometry
\end{abstract}

$\mathrm{G}$ as chromatography combined with mass spectrometry (GC-MS) provides analytical information that is definitive for many types of samples containing organic analytes. Over the last decade, fieldportable GC-MS systems have played a major role in key environmental as well as forensic applications [1-4]. The GC-MS technique provides conclusive and defensible information. Other field instrumentation provides information that can help to narrow an analytical focus; however, many lack the higher level of certainty inherent in the GC-MS analysis methodology. Field-portable GC-MS systems are widely used in situations that require rapid identification of the analyte(s) and a high degree of certainty in the data. For the Federal Bureau of Investigation (FBI)'s Laboratory Division, these goals are exacerbated when analysts are inundated with evidence as in the case of a downed airplane as shown in Figure 1. A strategy for handling evidence is often dependent on what scene investigators suspect. If it can rapidly be determined what chemical(s) are implicated, the evidence collection or field analyses can proceed more confidently. For environmental phenomena, such as shown in Figure 2, a field-portable GC-MS can be deployed to determine the chemicals present because of natural causes. The GC-MS can also be used to determine the time frame over which these chemicals appear (or disappear) so

\footnotetext{
Published online May 1, 2001

Address reprint requests to B. A. Eckenrode, Building 12, Room 327, Forensic Science Research Unit, Federal Bureau of Investigation, Quantico, VA 22135. E-mail: baeckenrode@fbiacademy.edu

This article was presented at the 12th ASMS Sanibel Conference on Field-Portable and Miniature Mass Spectrometry, Sanibel Island, FL, January $22-25,2000$.
}

that a correlation with other events can be established, such as an industrial release or natural fires. In situations where an incident has already been contained, an assessment of the chemical dangers present has to be made before an investigation can proceed. In these cases, the safety of personnel is paramount, and a GC-MS instrument on-site can be a valuable tool.

Field analysis requirements typically include the need for rapid assessment of the volatile organic compounds (VOCs) present, because these compounds can be inhaled and, therefore, represent the highest immediate health danger. The VOCs that represent the most credible and immediate danger to individuals include many industrial compounds, such as phosgene or cyanogen chloride, as well as chemical warfare agents (CWAs). Phosgene (a colorless gas) or the moderately volatile nerve agent Sarin (a colorless liquid with a vapor pressure of $\sim 22,000 \mathrm{mg} / \mathrm{m}^{3}$ at $25^{\circ} \mathrm{C}$ ) can be used to provide maximum and immediate effectiveness for a terrorist group. A test of water supplies to determine the level and distribution of contamination is less critical in terms of time than with VOCs; however, a liquid analysis may be required in instances where the chemicals used in a clandestine laboratory or found at the scene of a hazardous waste spill need to be rapidly determined for evidentiary reasons. When CWAs are suspected in a water supply, the need for rapid on-site accurate analysis is clearly evident. Soil or solids media require testing, generally with the lowest level of immediacy; however, if chemical warfare agents are suspected to be involved, the situation can be rapidly elevated in priority.

The detection and identification of chemicals used in 


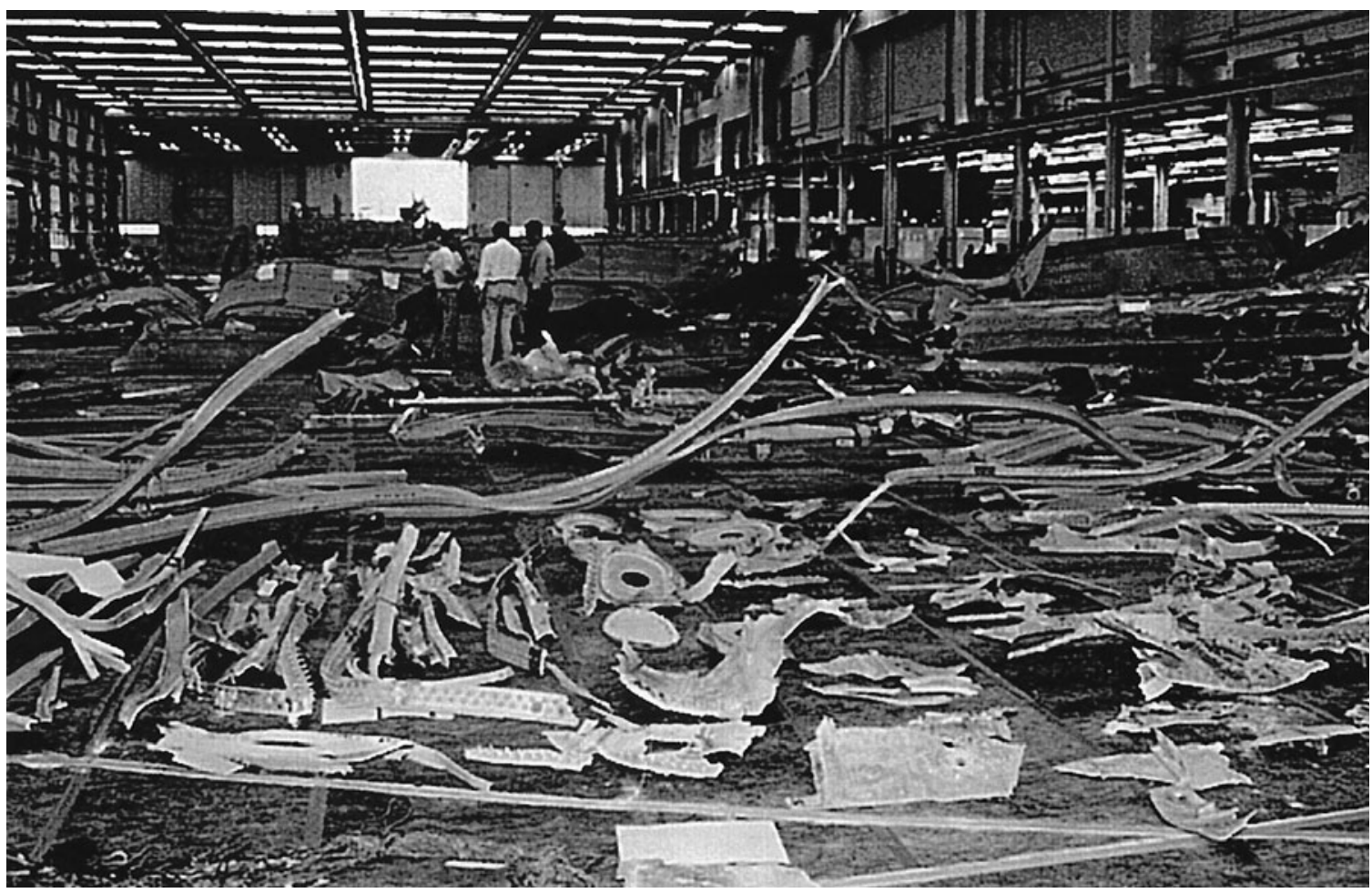

Figure 1. Field screening and/or GC-MS confirmational analysis can help address situations that require highly reliable and accurate results in a short time for hundreds of samples. Determining the cause(s) of an airplane crash can be a daunting analytical challenge and illustrates the need for lab-quality field instrumentation.

a deliberate release situation, especially when CWAs may be involved, can be much more difficult than what responders encounter during a hazardous spill due to a transportation accident or a release from an industrial incident. In many accidental cases, responders can make judgments based on labels, posted warnings, or material safety data sheets (MSDs); however, with the investigation of a deliberate organic chemical release the situation is far worse, and therefore, the analysis tools available become more critical. A few types of analysis systems typically employed for unknown field vapor analysis situations include the following:

- photoionization detectors (PIDs) with and without a GC

- electrochemical and catalytic bead gas detection systems

- colorimetric tube systems

- card kits using liquid chemical reagents

- ion mobility based systems (IMS)

- GC-MS

Each of the detection systems or methods listed above has unique strengths; however, the GC-MS methodology offers the best collective analytical capability. GC-MS combines the high sensitivity of an IMS, the dynamic range of a PID or a flame ionization detector (FID), the specificity of a gas detection system, tube system, or card kit, and adds the selectivity required for rapid identification of many organic compounds. For example, most organic compounds have ionization potentials less than $10 \mathrm{eV}$ and screening with PIDs is possible. Problems arise when these instruments cannot differentiate between hazardous materials and other ionizable chemical vapor. Problems also arise when there are cross-sensitivities or interferences such as insect repellent or burning gasoline. In most cases, a confirmatory method is desirable. A field-portable GC-MS can complement scene investigations by providing an on-site confirmation of the results generated by other screening tools.

There are many instances where field GC-MS systems have been deployed to address issues that require rapid answers and reliable conclusive information. The primary focus of this special focus paper concerns a GC-MS system that has been used for investigative VOC measurements in a harsh jungle environment. This paper also discusses the use of field GC-MS in a process chemical demilitarization scenario and in a military installation for mobile real-time detection of deleterious organic vapors.

\section{Experimental}

\section{Environmental Analysis-Jungle Application}

The Viking model 572 field-transportable GC-MS instrument (newer models are currently produced by 


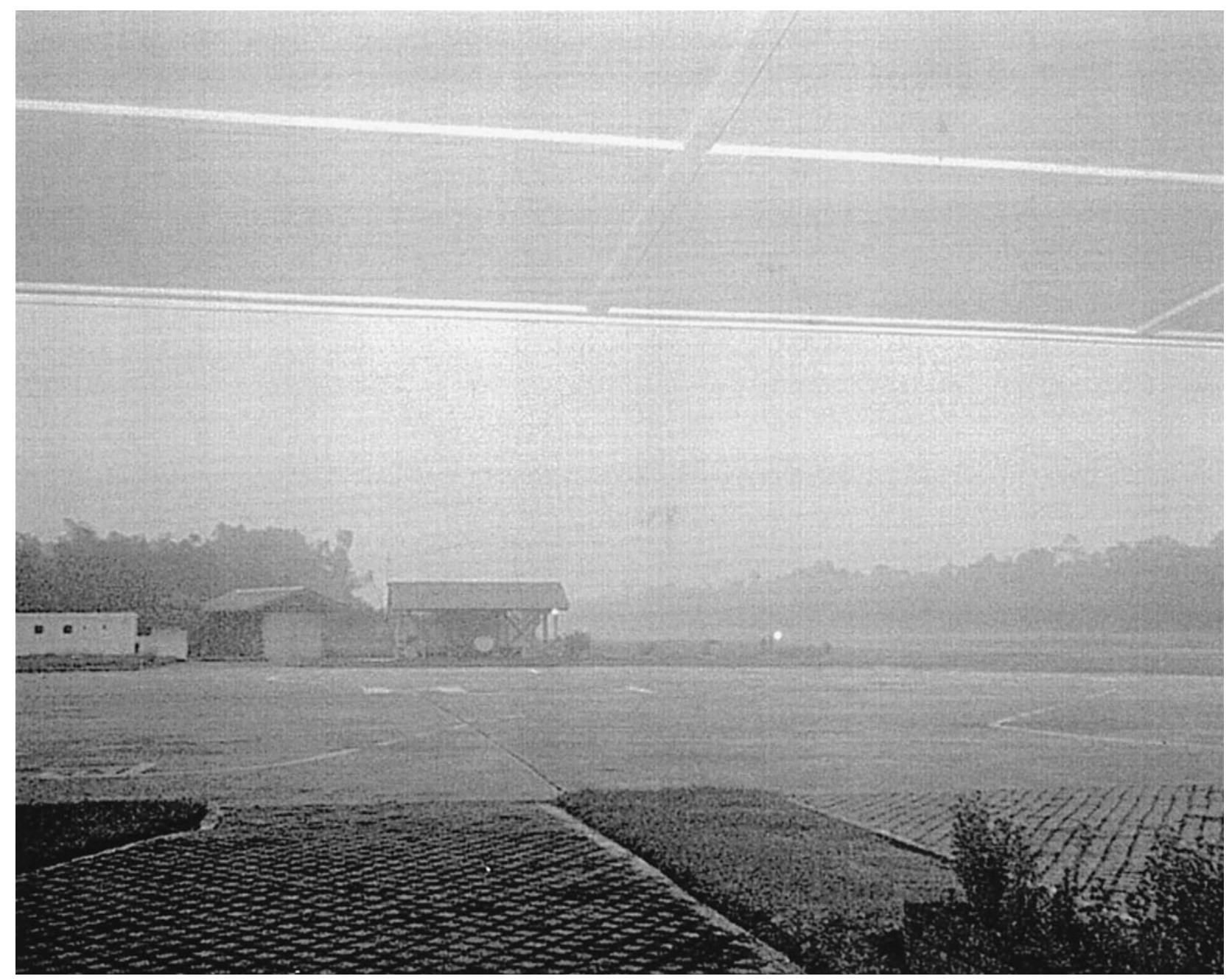

Figure 2. The haze generated by naturally occurring fires due to a severe drought in Malaysia settles over the Lutong airstrip in early morning. The bright spot to the right of center is the retro-unit for the long-path reflective FT-IR system used in conjunction with the GC-MS field instrument.

Bruker Daltonics, Inc.) was used for many of the analyses and is detailed elsewhere [2]. This field-proven GC-MS system was placed in two cargo cases and shipped to the city of Miri on the island of Borneo in Malaysia to investigate the cause of a refinery incident involving the destruction of an air separation unit. Initial tuning of the instrument to the client's protocol was performed in a controlled setting before placing the GC-MS into a locally rented van that was additionally outfitted with a long-path reflective infrared system. The rough terrain and the dusty environment were particularly challenging and tested the design and field-readiness of the GC-MS; however, the GC-MS functioned flawlessly throughout the entire three-day intensive on-site VOC data collection. Over the course of these field measurements, the ambient temperature varied from $27.5^{\circ} \mathrm{C}$ to $30^{\circ} \mathrm{C}$, and the relative humidity varied from $76-80 \%$. When mobilized throughout the jungle, as shown in Figure 3, a portable $2 \mathrm{KW}$ power generator was used for both of the detection and analysis systems. When positioned at a local unused airport, site power was utilized.

The integrated inlet valving and control system utilized for this project is shown in Figure 4. A Tenax (Enka Research Institute, Arnhem) fixed trap was used as a VOCs concentrator with a $20 \mathrm{~m}, 0.18 \mathrm{~mm}$ id., 1.7 $\mu \mathrm{m}$ film thickness, VRX column (Restek Corp., Bellefonte, PA). Outside air entered the fixed trap inlet of the instrument via a 10-m Teflon (E.I. duPont de Nemours \& Co., Inc.) tube with an inner diameter of 6 $\mathrm{mm}$ using the instrument's on-board sample pump. Typical air volumes measured with an on-board mass flow meter varied from 0.5 to $2.5 \mathrm{~L}$ for the different on-site air sampling experiments. After the integrated analyte concentrator trap was loaded at ambient temperature, a $0.5 \mathrm{~min}$ helium dry purge was used to reduce the moisture and $\mathrm{CO}_{2}$ contribution prior to preheating. The trap was subsequently preheated to $210{ }^{\circ} \mathrm{C}$ for 0.2 minutes and desorbed at $240{ }^{\circ} \mathrm{C}$ for 4 minutes. The GC initial temperature was held at $35^{\circ} \mathrm{C}$ 


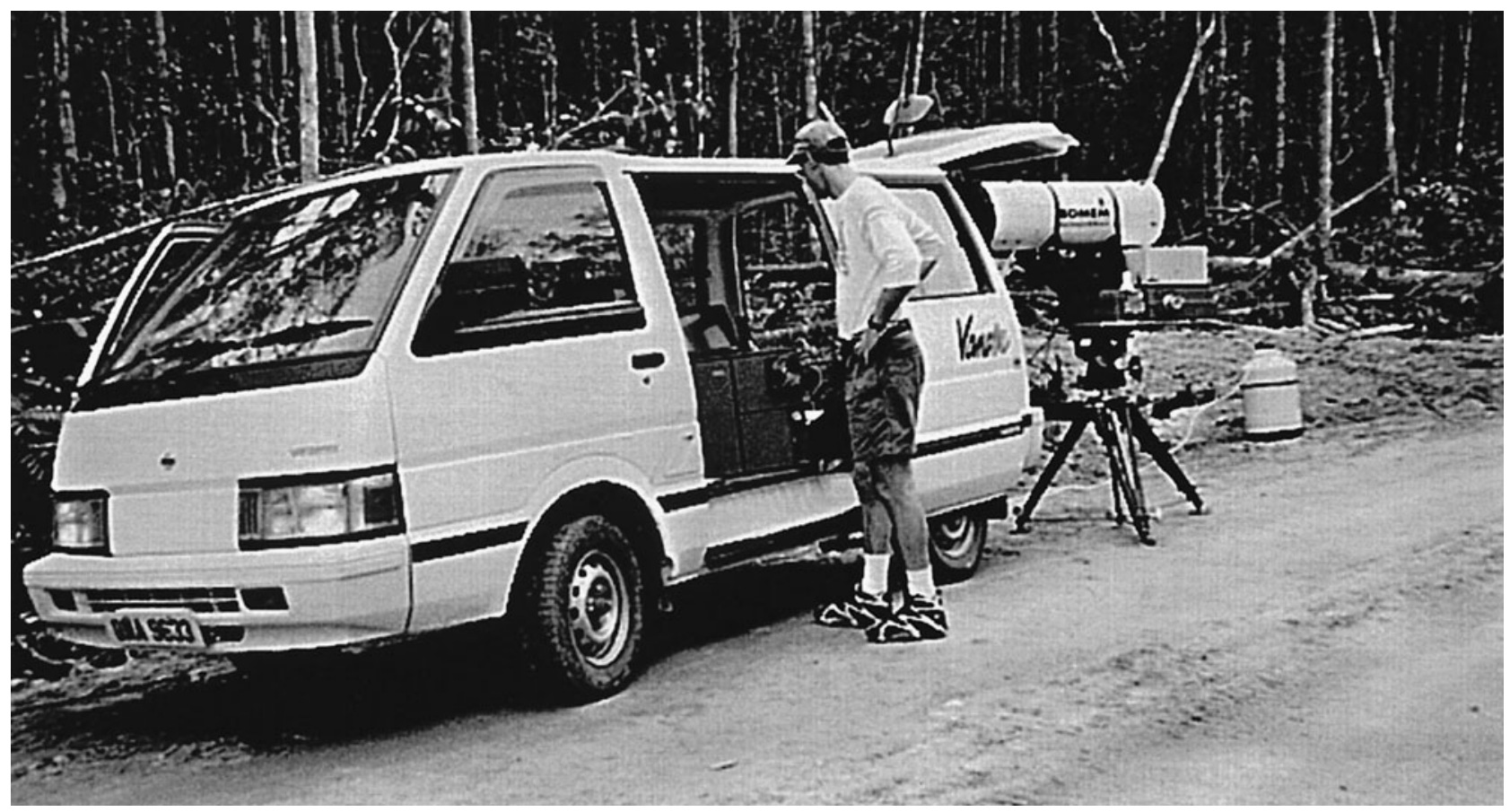

Figure 3. Volatile organics analysis in the Malaysian jungle with a transportable GC-MS and a transportable long-path reflective FT-IR.

for 1 minute and was ramped at $10^{\circ} \mathrm{C} /$ minute to $200^{\circ} \mathrm{C}$ with a hold of 0.5 minutes. The total run time was 18 min and the MS was scanned from 35 to $350 \mathrm{~m} / \mathrm{z}$. A liquid standard of aromatic and alkane compounds at $50 \mathrm{ppm}_{\mathrm{w}}$ was used to provide a single-point on-site calibration for semiquantitative measurements of the contaminants.

An unused airstrip at Lutong provided shelter and power for the GC-MS, and it was here that in addition to several individual experiments, a continuous unattended 12-hour air sampling and analysis was performed. Trap blanks were performed under identical desorption conditions both pre- and post-continuous analysis data set indicating nondetectable carryover at these sample volumes.

\section{Forensics-Chemical Demilitarization and Military Vehicle-Mounted GC-MS}

The Viking model 772 GC-MS, (designed for process applications and currently produced by Bruker Daltonics, Inc.), was integrated into a process trailer designed and constructed for chemical demilitarization purposes. This model was constructed around the model 572 GC-MS; however, it differs in several respects. The model 772 GC-MS can be remotely controlled to collect and analyze the sample vapor emanating from or generated in a munitions treatment vessel (MTV) where a munition can be mechanically breached. A block diagram showing how the GC-MS system integrates with the other essential elements in the munitions breach and treatment is shown in Figure 5. A major goal for the GC-MS system is to confirm the results of the GC-based analyte specific monitors (MINICAMS, CMS Field Products, Birmingham, AL) being used for detection, but with limited identification capability.

This process GC-MS is further differentiated from the model 572 system by its uniquely packaged inlet design along with a specific data, remote control, and communication computer system. A picture of the system is shown in Figure 6. A heated $10 \mathrm{~mL}$ nickel 200 gas loop (held at $175^{\circ} \mathrm{C}$ ) mode allows sampling of the vapors emanating from within the MTV when the expected concentrations may be relatively high, in the $\mathrm{ppm}_{\mathrm{v}}$ range.

Another fieldable GC-MS system similar to the process GC-MS, called the Chemical Agent Detection and Identification System (CADIS), has been evolving over the past several years [4-6]. Currently, this military vehicle-mounted GC-MS has a more versatile vapor sample inlet. A picture of the military-hardened analyzer is shown in Figure 7, and a schematic of the inlet valving is shown in Figure 8. This inlet is designed around an eight-position software-controlled stream select valve. The vapor sample is pulled into the vehicle by a high-speed sampling blower working in conjunction with a Cyclone (GO Regulator Inc., Willow Grove, PA) filter using 10 micron porosity particulate filters. The main flow of 1-3 L/min, provided by the Cyclone filter and blower combination, minimizes both the sample transport lag time as well as the sample line losses. This main flow is "sampled" by the membrane or fixed trap inlet of the Viking model 572 GC-MS (integrated into 


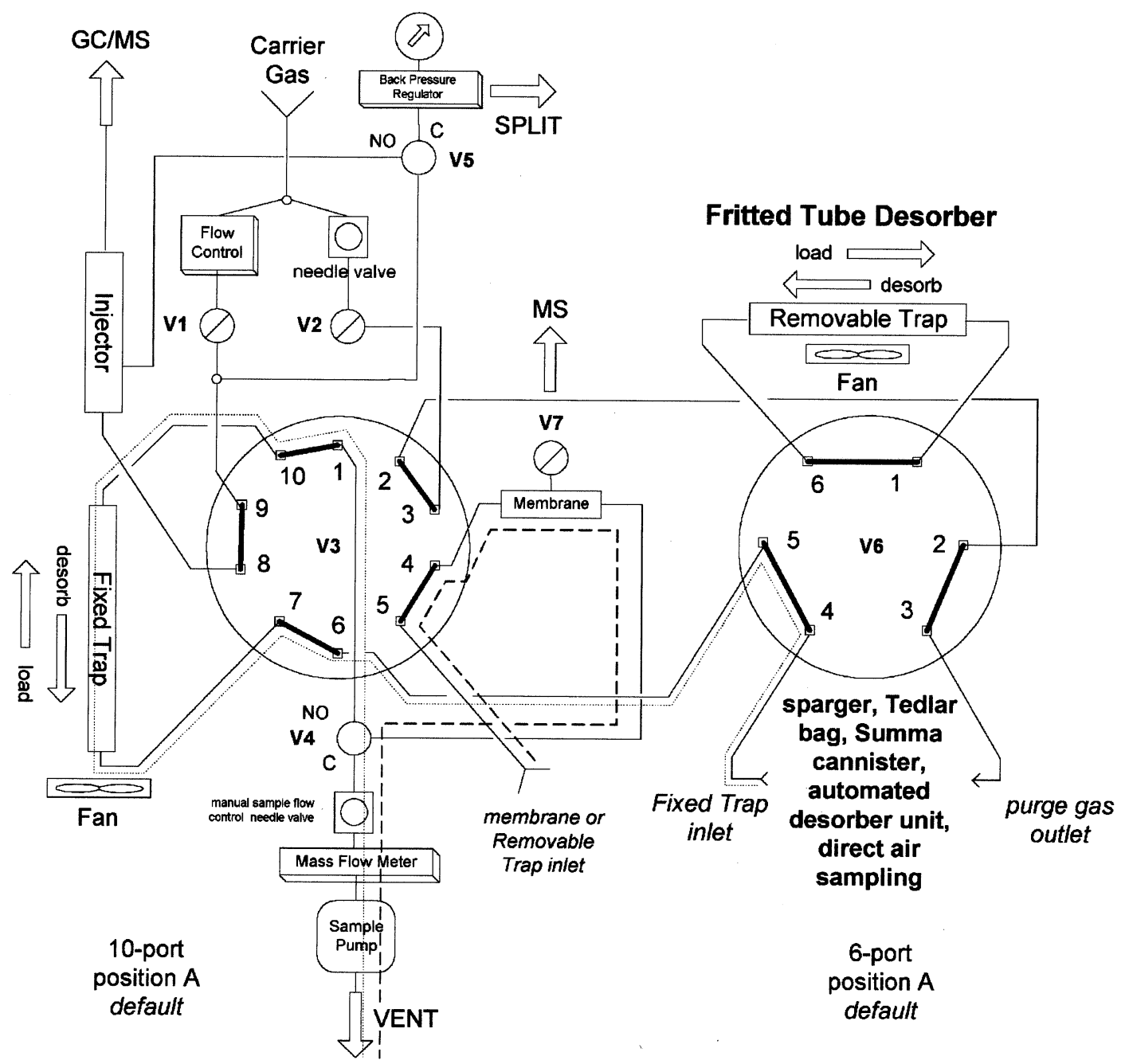

Figure 4. A detailed inlet diagram of the valving and flow paths for the trap-load (dotted) and membrane inlet (dashed) sampling cycles. The membrane can be used for rapid screening with MS detection, and the trap can be used for concentrating the VOCs followed by rapid desorption and GC-MS.

the CADIS) at a rate of approximately $100 \mathrm{~mL} / \mathrm{min}$. Due to the low volatility of the nerve agent VX, a VX-to-G-analog conversion pad of silver fluoride can be inserted into the feed line between the Cylcone filter assembly and the GC-MS, as illustrated in Figures 8 and 12. Because this form is similar to the chemical warfare agent Sarin (a G-type agent-GB), the product of the conversion reaction is termed the G-analog. The additional blower in this valving arrangement uses the common outlet of the stream select valve to purge the other sampling ports simultaneously during sample acquisition. The CADIS also has a specially designed air sampling head and an electrically controlled surface probe. The surface probe is used only when the vehicle is motionless. The probe's head is used to heat the surface of the soil and pull the outgassed vapors into the Cyclone filter and subsequently to the analyzer.

\section{Results and Discussion}

\section{Environmental Application}

In the late spring to early summer of 1998, a European chemical company wanted to determine the identification of the organic compounds present in the haze generated by the drought and subsequent fires in the jungles of Malaysia (see Figure 2). The company was concerned that some of these compounds may interfere with well-established and properly functioning industrial reaction processes that they were operating on the island of Borneo. They were not sure how localized the haze was and sampling around the facility and in the nearby jungle was required. This made sorption tube sample acquisitions in many cases impractical; however, with the addition of a mobile GC-MS some measurements could be made relatively quickly at various points around their air separation unit. The 


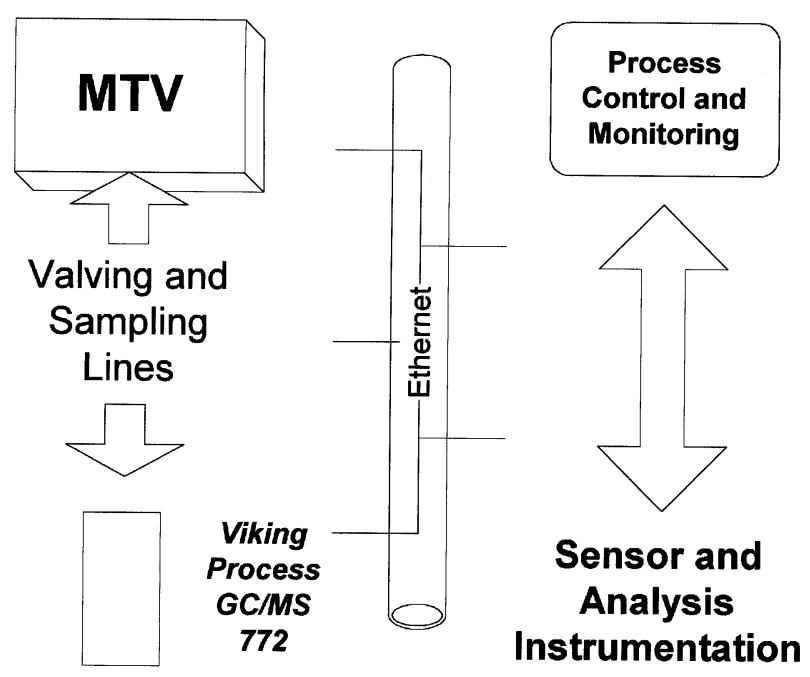

Process Trailer

Mobile Unit

Figure 5. A block diagram showing how the GC-MS integrates into the MMD1 facility. The Bruker/Viking 772 GC-MS located in the process trailer samples the munitions treatment vessel (MTV) organics and relays the information to the control trailer system via an ethernet link.

immediate data generated by the mobile GC-MS could help define proper locations for more extensive studies with particulate analyzers and sorption traps. Also, because the haze could contain a variety of organic compounds and they wanted a real-time on-site contaminant assessment, a transportable GC-MS was selected as the analytical instrument of choice for this short-term analysis project.

Throughout the day on the Borneo coastline, the visible haze was not very low to the ground and was more disperse; however, at other times the haze was nearly opaque. Fortunately, this transportable GC-MS system has the ability for unattended operation over several hours so that this environmental inversion could be studied with respect to the haze contaminants. A determination of the highest concentration of contaminants could be measured in the morning, and the levels decreased throughout the day. A slight increase in the haze thickness can be observed and measured between the hours of 5 and 8 p.m. The vapor phase contaminants were found to consist primarily of $C_{3}-C_{21}$ aliphatic hydrocarbons at high $\mathrm{ppm}_{\mathrm{w}}$ levels, with lower $\mathrm{ppm}_{\mathrm{w}}$ levels of terpenes, aromatic hydrocarbons, and benzene including alkyl substituted species. The reconstructed total ion chromatograms for an analysis early in the day and a nighttime analysis are shown in Figures $9 a$ and $b$, respectively.

Although most of the data was considered proprietary, an example of the data obtained for one particular organic analyte found in the haze, a plot of the ion current area of dimethyl sulfone versus time, is shown

in Figure 10. It is apparent that the dimethyl sulfone levels are tracking the coastal inversion process which is observed to occur naturally. Although dimethyl sulfone was generated by the fires to a slightly greater degree than the other detected compounds, its presence can be partially explained by the heavy sulfur odors and rich organic peat that was sm oldering and stripping the organic compounds out of the jungle plant life as the combustion process continued throughout the day and night. Nevertheless, these data allowed the investigators to begin experimentation with the major classes of compounds measured in the haze to observe how other chemical systems on the island may be affected.

\section{Forensic Applications}

A project to develop a mobile munitions management device (MMD) to first characterize and subsequently destroy non-explosively configured non-stockpile chemical weapons, was directed by Congress and initiated several years ago by the U.S. Army [7]. A highly engineered facility in terms of personnel safety and munitions destruction effectiveness was designed and constructed, containing analytical test equipment specifically configured to detect and identify select chemical warfare agents (CWA). Several experiments were conducted to assess the ability of the well-equipped process trailer to measure known CWAs and industrial gases generated from various locations within the trailer. Measurements were made from the distal end (the MTV) and at various points throughout the $15 \mathrm{~m}$ path to the GC-MS. Figure 11 shows an example of a phosgene analysis subsequent to a $2.7 \mathrm{ppm}_{\mathrm{v}}$ release into the remotely located munitions treatment vessel (MTV) and analyzed by the GC-MS operating in the selected ion monitoring (SIM) mode. All of the sample vapors are directionally controlled near the MTV by joint and concurrent signals from the GC-MS and the separate control trailer. Before any measurements are made with the GC-MS, both the process trailer and the control trailer must activate the appropriate valving.

When the integrated adsorbent bed is used, followed by a subsequent desorption step to the GC, the analyte concentrations are typically very low in the MTV. This methodology can be used when sampling highly toxic CWAs such as VX or its fluorinated analog (G-agent analog). A silver fluoride pad was added to a $10 \mathrm{ppm}_{\mathrm{w}}$ solution of VX in isopropyl alcohol and the derivatization reaction to the more volatile analog, was allowed to occur for five min prior to injecting $2 \mu \mathrm{L}$ of the solution into the heated lines at the MTV. The results from the vapor concentration and desorption analysis after the sample had passed through the sample lines from the MTV to the GC-MS, are illustrated in Figures 12a and b. The mass chromatograms obtained from the full scan data are in the proper ratios with the ion at $m / z 111$, providing an added 


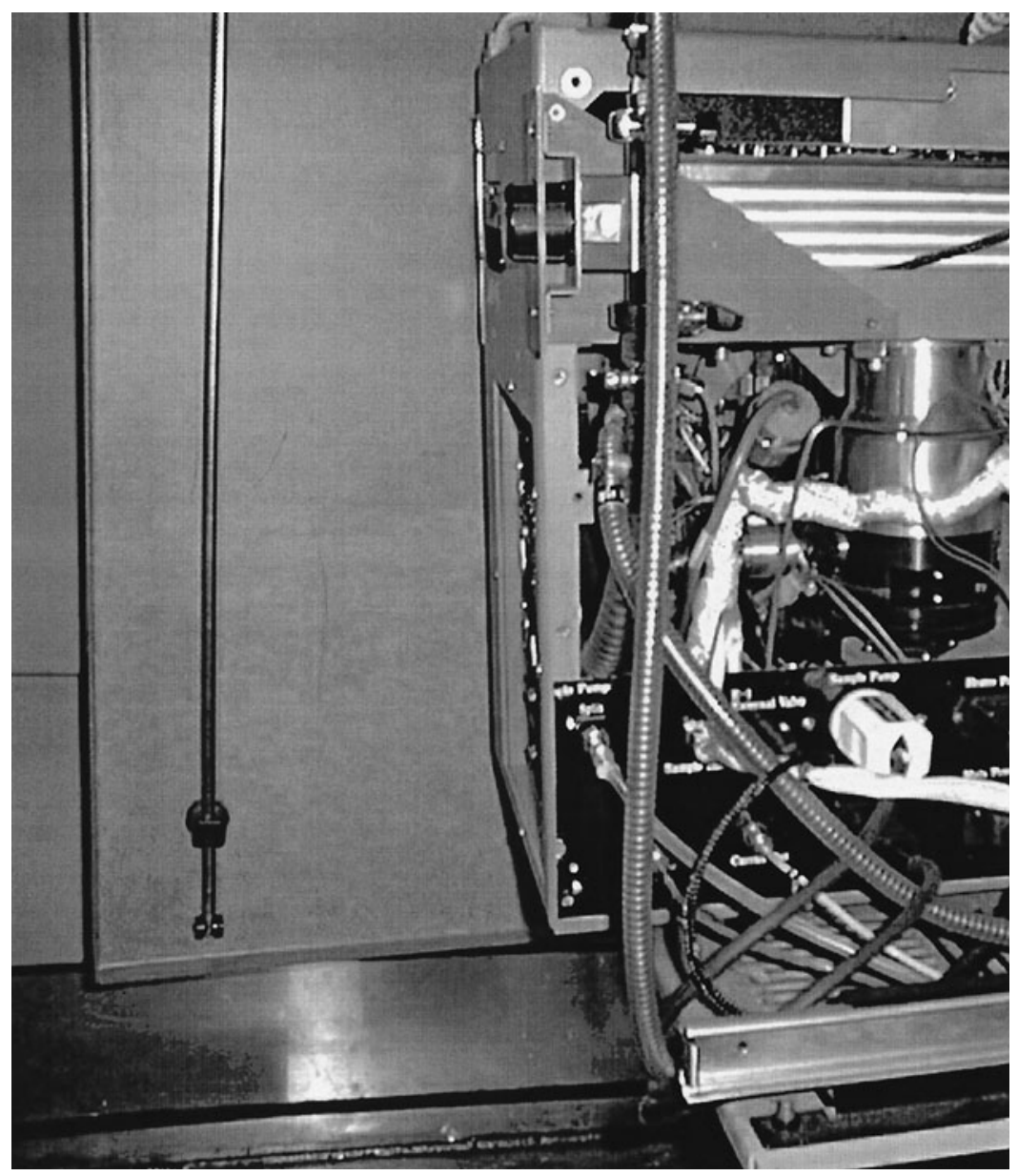

Figure 6. A picture of the process GC-MS shown within its NEMA enclosure inside the process trailer of the MMD1 facility.

differentiating feature from the other CWAs, including the nerve agent Sarin (GB).

\section{Forensics-CWA Analysis via Military Vehicle- Mounted GC-MS}

With chemical reconnaissance, detection and warning are essential elements of chemical exposure avoidance. Although different types of equipment for CWA detection exist, few systems can provide both good sensitivity and selectivity. During the Gulf War, several warning alarms were reported; however, some of these alarms still cannot be explained or confirmed [8]. In the military system described above (see Experimental), the GC-MS is designed to provide rapid screening data via membrane inlet mass spectrometry prior to confirmation tests via GC-MS, when necessary.

Major features of the CADIS include the capability to acquire VOC data, perform a detection and identification algorithm, and trigger an alarm (if warranted) while moving at a relatively high rate of speed. When a signal above a pre-set threshold and the data system analysis algorithm trigger an alarm for an analyte, the vehicle is directed to stop for further sampling and confirmational testing. The membrane inlet mass spectrometry (MIMS) operational cycle can detect $\mathrm{ppb}_{\mathrm{v}}$ levels when the system is operated in SIM mode. Subsequent samples collected while the vehicle is stopped are typically of greater volume (as vapor) over the specific area so that the analyte(s) can be concentrated in the adsorbent bed and full mass spectral scanning can then be employed as well.

\section{Real-Time MS Detection and Identification Algorithm}

Targeted analyte deconvolution algorithms have been developed for post-processing GC-MS data [9]; however, real-time MS algorithms of VOCs are in the formative stages. Collection of the MS data via MIMS with the CADIS while the military vehicle is moving requires an intelligent real-time data analysis approach. A real-time SIM-based algorithm was designed by Viking Instruments Corp. (currently owned by Bruker Daltonics, Inc.) scientists and tested to measure CW 


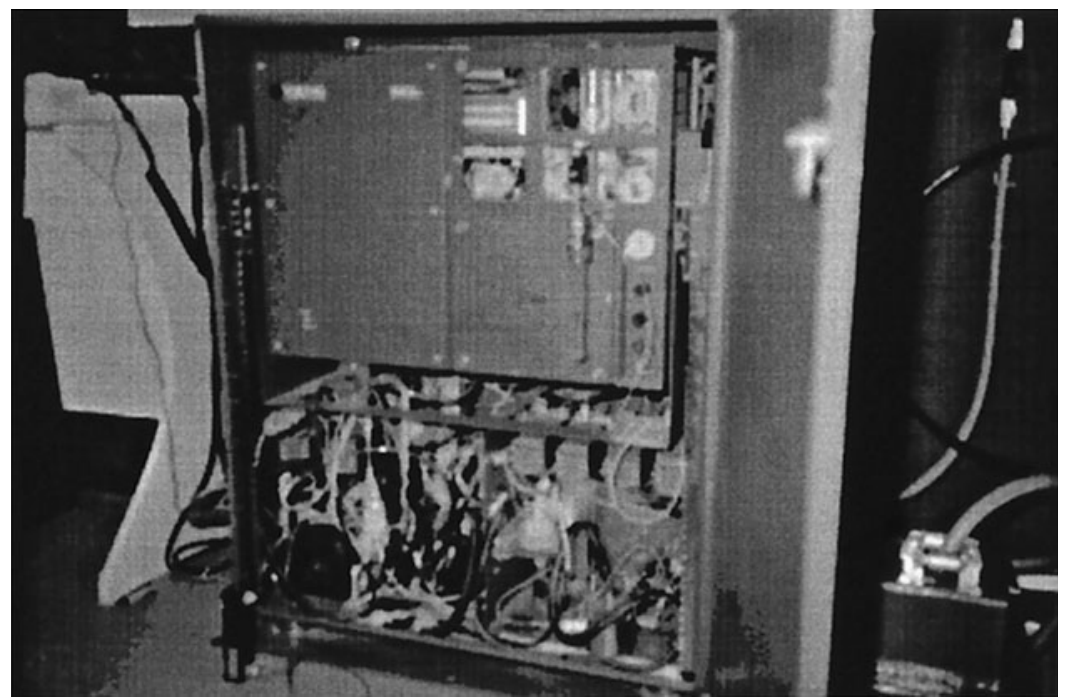

Figure 7. A picture of the CADIS within its shock-resistant housing. The surface probe exits the floor (lower right) and the air sampling line exits the roof (upper right) of this reconaissance military vehicle.

simulants. An overview of the ten-step algorithm is provided here.

1. Determine if the instrument is functioning. Measure the abundance of a unique ion such as Argon at $\mathrm{m} / \mathrm{z}$ 40 and if it falls within a user selectable range, then continue with the acquistions; otherwise, warn the user.

2. Pre-filter the data. Using an estimated total ion current (TIC) threshold for the base ion or a ratio of abundances (based on prior empirical measurements), measure the TIC for each targeted analyte. If the threshold for the appropriate ions is less than the pre-set threshold, then exclude the analyte from further measurements during the sampling interval.

3. Normalize the scan data. The normalized ratios are used over the SIM time (scan) interval.

4. Compute the difference matrix. This is the difference between a row vector containing all abundance

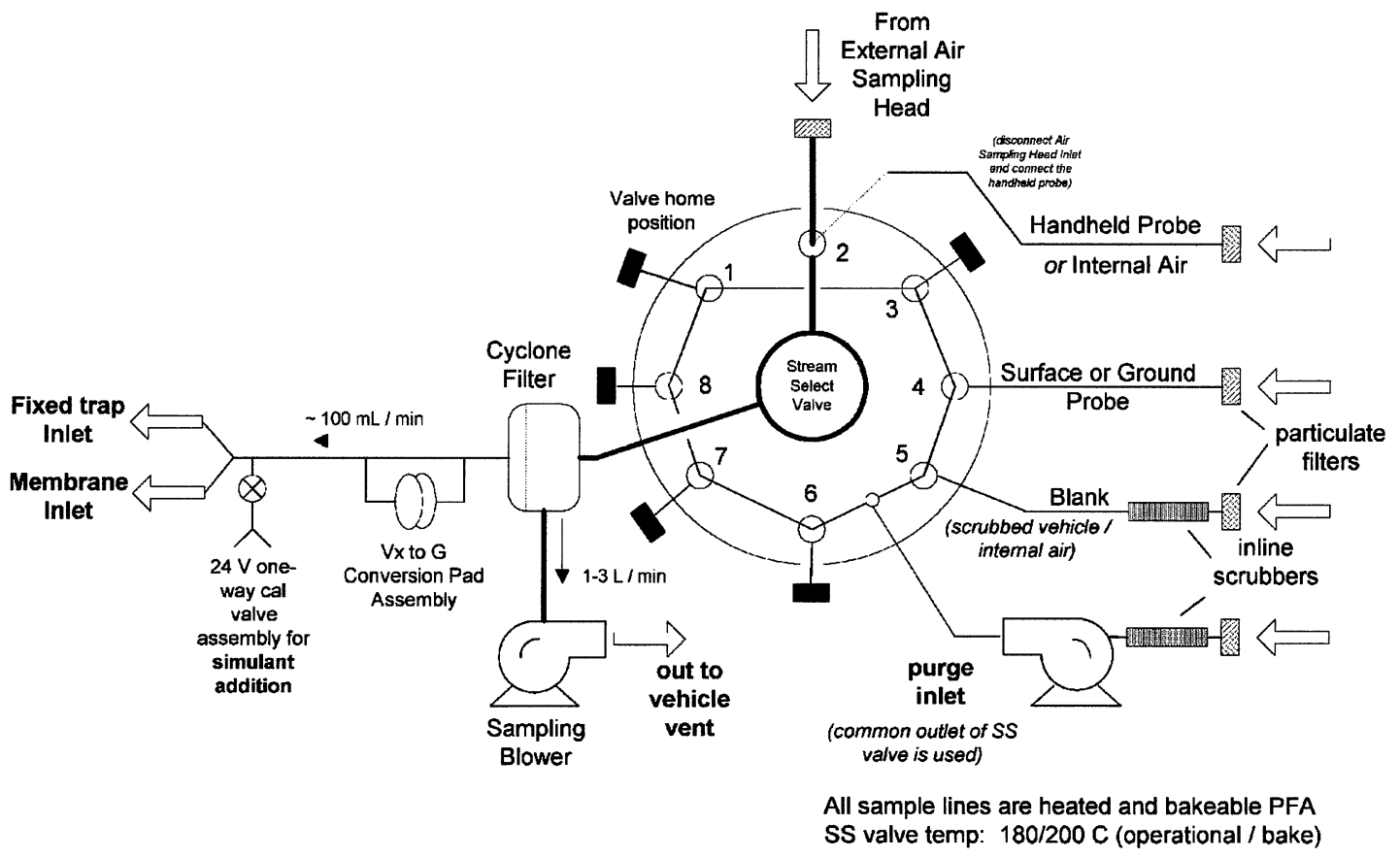

Figure 8. The CADIS inlet valving is designed around a heated stream-select valve, a Cyclone filter, and dual inlet/outlet blowers. This valving is ahead of the valving shown previously in Figure 4, and acts as a front-end for the trap or the membrane inlets of the analyzer. 

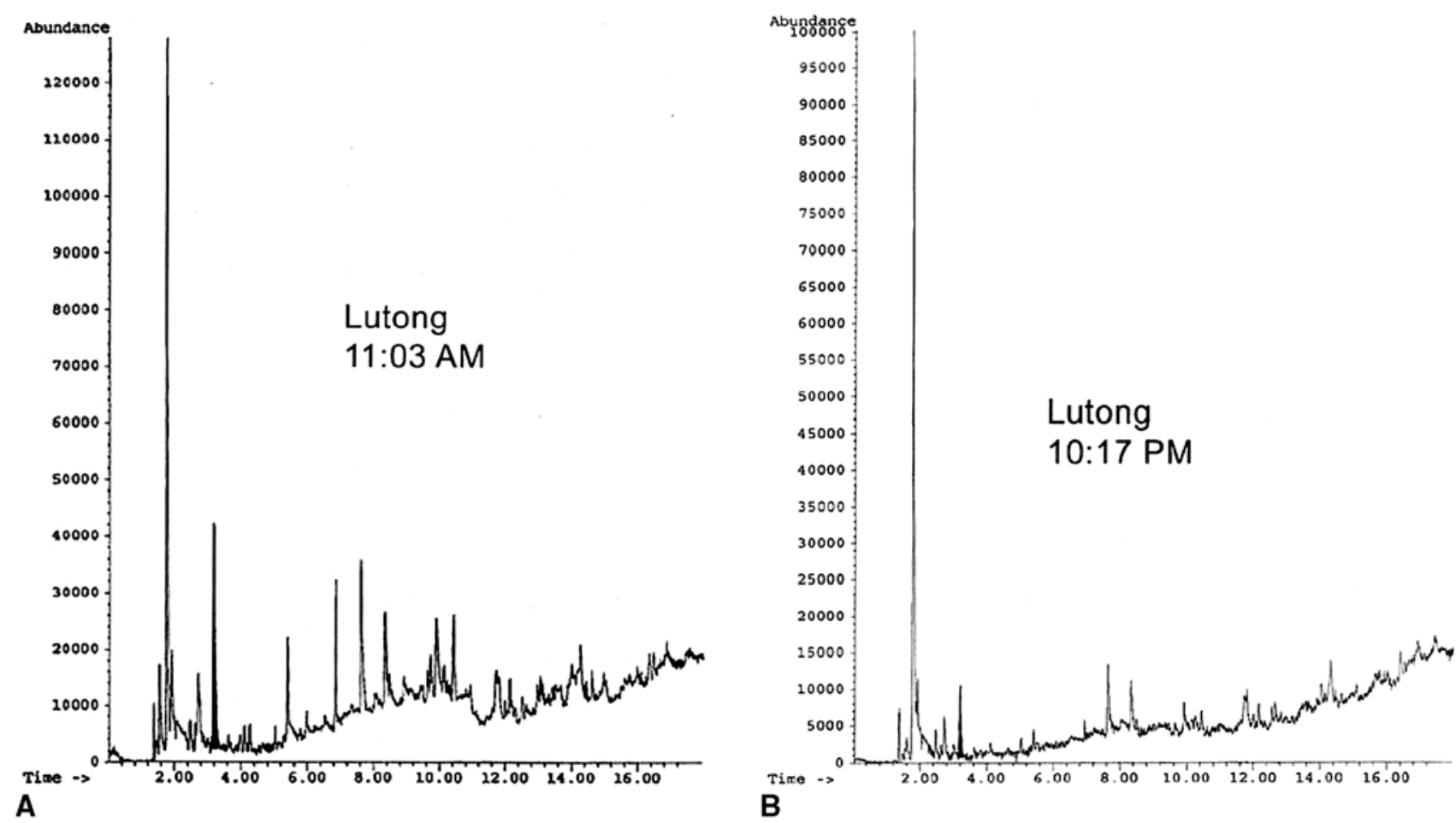

Figure 9. Selected total ion chromatograms (TIC) from an unattended 12-hour repetitive analyses (trap-and-desorb) of the VOCs at the airstrip in Lutong on the island of Borneo. The TIC in (a) was acquired in the morning and the TIC in (b) was acquired in the late evening. The dimethyl sulfone peak is shown filled in at a retention time of $3.2 \mathrm{~min}$.

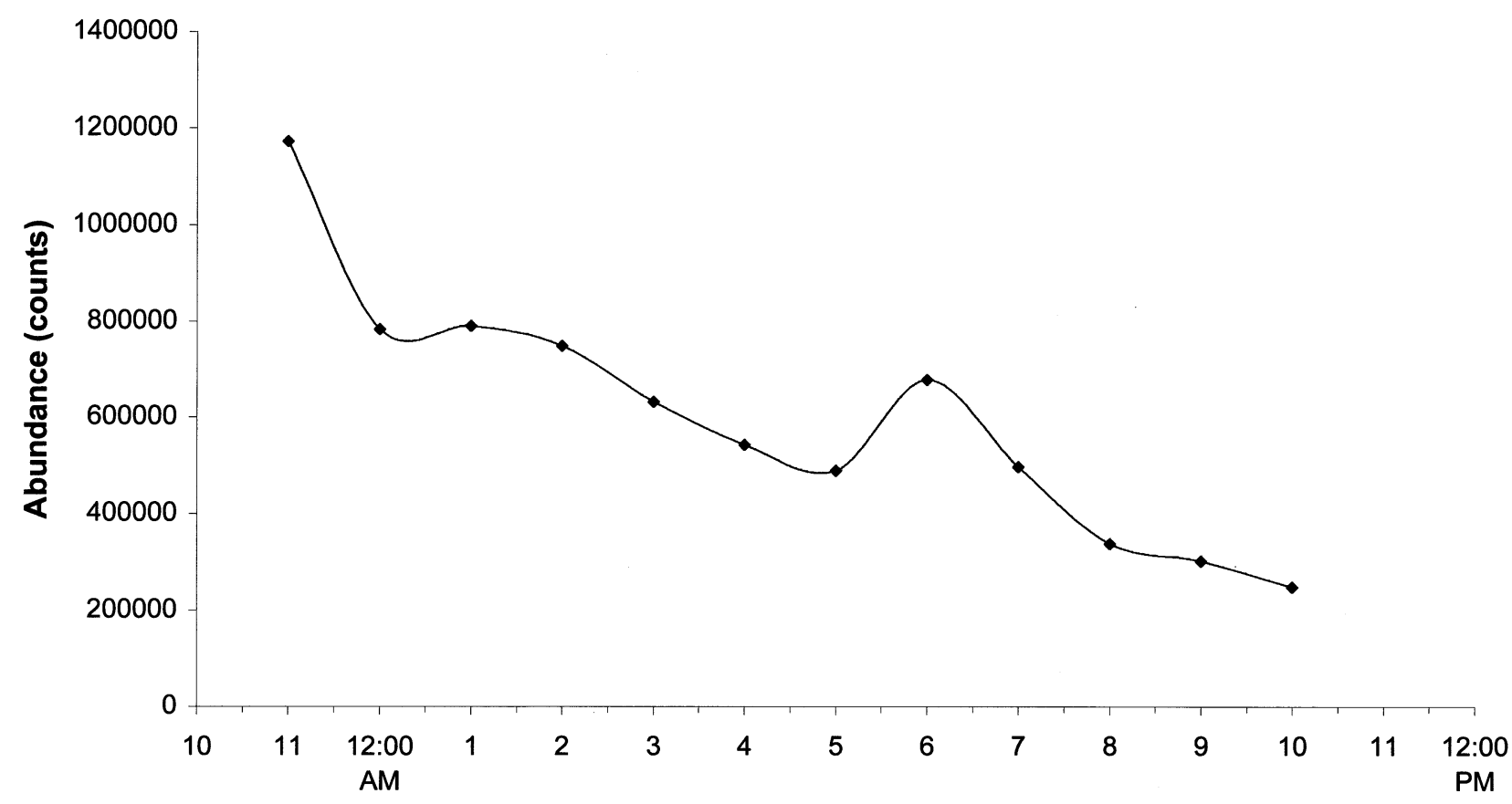

Time (hours)

Figure 10. A plot of dimethyl sulfone area counts versus time over the course of a 12-hour period on the island. These data captured the fluctuations of the various organic compounds generated by the drought and subsequent fires in Malaysia. 


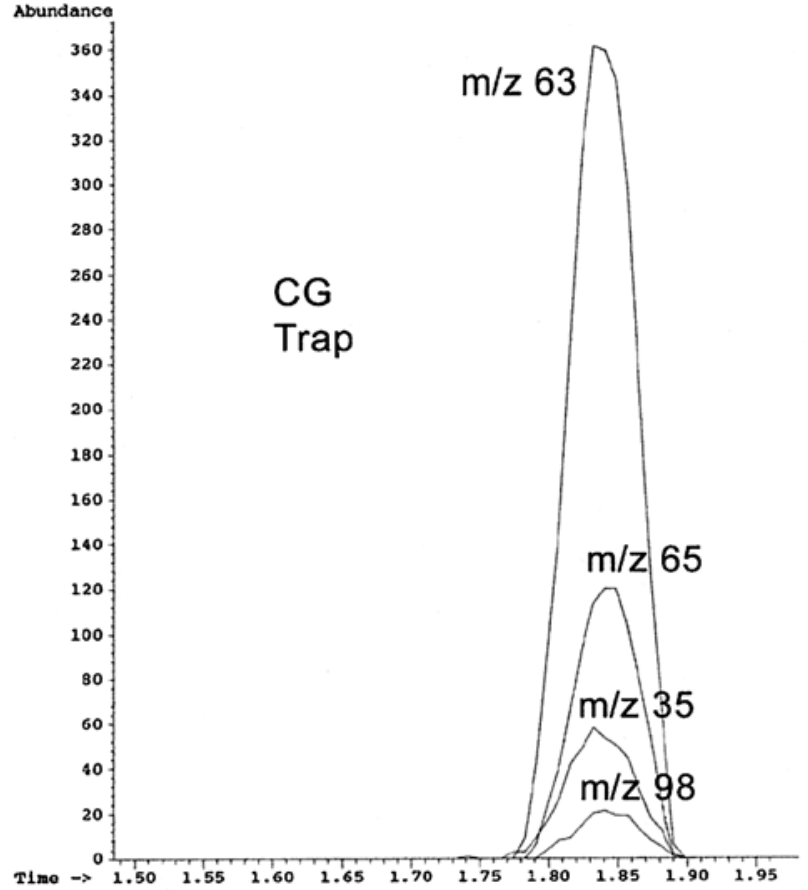

Figure 11. The selected ion monitoring results of a $2.7 \mathrm{ppm}_{\mathrm{v}}$ release of phosgene (CG) into the MTV measured with the process GC-MS. The fixed trap was loaded for $5 \mathrm{~min}$. at $80 \mathrm{~mL} / \mathrm{min}$. with a 0.2 min dry purge.

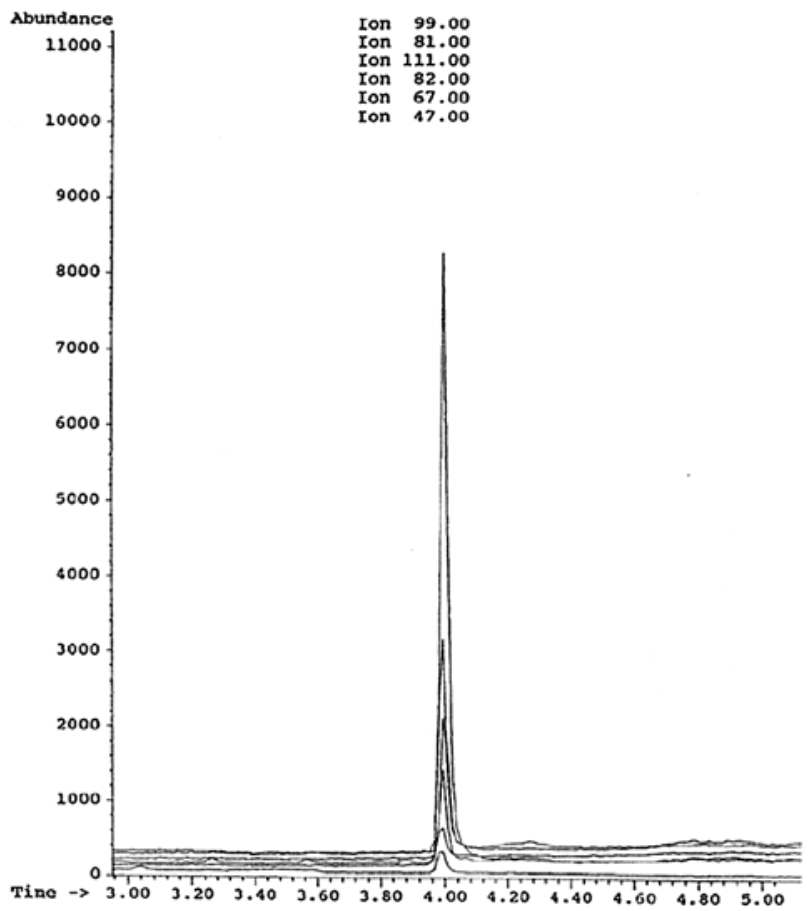

A values as a pre-defined pattern (model) and the matrix containing all abundance values of a particular data file (differentiated by time).

5. Weigh the difference. Empirically adjust for select analytes a unique response function.

6. Further weigh the differences. Based on the TIC from each SIM acquisition, changes over a settable time represent a normalized slope, i.e., a hit is more likely if this value is increasing and less likely if this value is decreasing.

7. Statistically test the difference. A chi-square statistic is used to determine the significance of the difference between the model and the scans. The confidence level is configurable.

8. Alarm/No Alarm. Sound an audible alarm within the vehicle if the data indicate that an agent may be present above a set confidence level. If the alarm sounds, the system will alert the operator to inform the driver to stop and begin the confirmation cycle.

9. Repeat for each agent. The model database contains up to 40 analytes with the option to select from 1 to 5 ions per analyte.

10. Repeat sampling interval (Loop).

This real-time algorithm is still in development and will require extensive testing; however, when combined with the modified inlet valving it provides the necessary data analysis system to assess and defend against potentially dangerous plumes that field personnel may

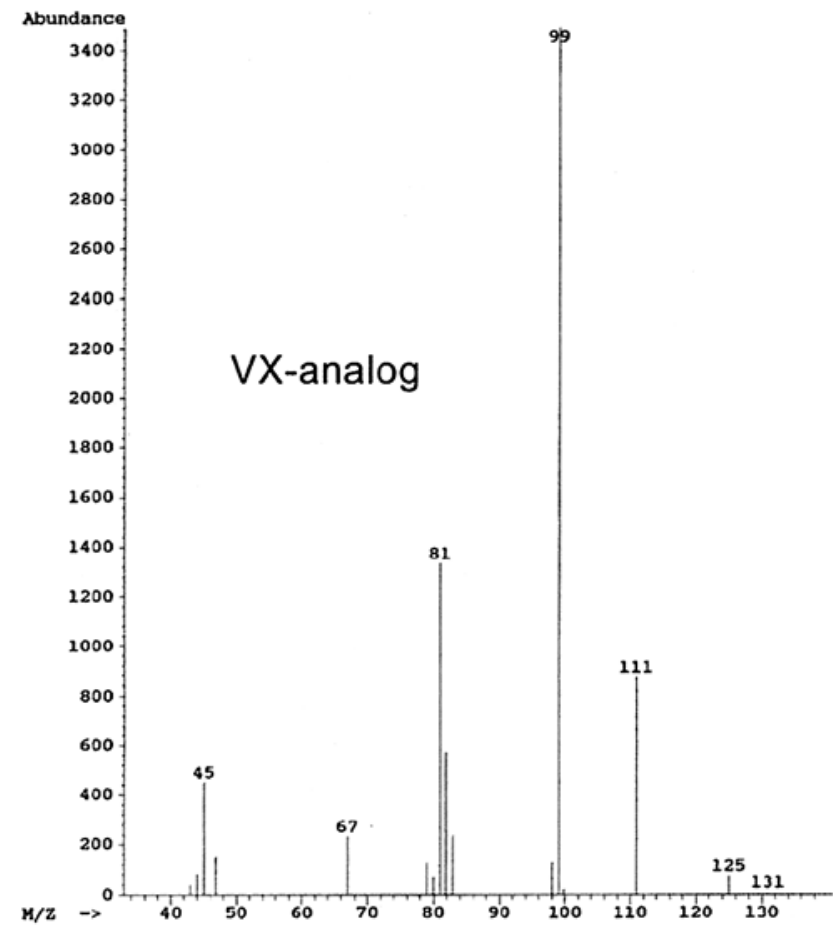

B

Figure 12. (a) Extracted ions from a full scan acquisition of the product from the derivatization reaction of O-ethyl-S-(2-isopropylaminoethyl)methyl phosphonothiolate (VX) with silver fluoride. (b) Mass spectrum of the more volatile G-agent analog (VX-to-G-analog) derivatization product with a characteristic ion at $m / z 111$. 
enter. False alarms can be problematic primarily because of the need to employ protective clothing when it is not necessary and to use a CW antidote may be forced. A major factor in this GC-MS system is the ability to measure sub- $\mathrm{ppb}_{\mathrm{v}}$ levels and thus, the probability of escaping an organic plume without serious health consequences remains high. The analyte confirmation aspect employing the GC (after a hit via MIMS) helps reduce the possibility of false alarms and additional health consequences that may result from the CWA antidotes themselves or the limitations imposed by protective gear. The drawback to the GC analysis approach is that it takes much longer relative to the membrane analysis.

\section{Conclusion}

The applications discussed above indicate that there is a need for transportable and portable instrumentation that is sensitive, selective, precise, and accurate. The objective is to provide decision-makers with the best data analytically possible. When organic chemicals are involved, GC-MS and MIMS ranks very high on the list of tools that many investigators would like to use. There remain many obstacles to overcome so that a greater community of users can adequately and economically deploy this type of instrumentation. This instrumentation is still bulky (vacuum system, etc.), power hungry, and somewhat fragile. The FBI supports development of the concept of a totally transportable laboratory (flyaway laboratory) [10]; however, it is clear that many of these systems are far from miniaturized. For the GC-MS systems, major developments have been made in reducing the size of the GC; however, many of these new GCs have either not demonstrated an ability to maintain laboratory-level performance or have not been successfully commercialized. One particular system developer has been able to keep conventional columns in use while dramatically reducing the power of a standard GC (to 1-5\%) and eliminating the oven [11]. The size of the MS has also been reduced, as we have witnessed at this Sanibel conference, with promising developments for future field instrumentation [12]. The Hapsite GC-MS (Inficon, East Syracuse, NY) has been developed primarily as a vapor analyzer and has proven to be quite effective in detecting organic compounds with a boiling point of less than $180{ }^{\circ} \mathrm{C}$. The Hapsite is battery powered and can be carried by responders into dangerous (hot) zones with the data subsequently downloaded to a laptop for analysis. It is also being modified to include a sorbent bed and a rampable GC requiring low power. Currently, the FBI's Hazardous Materials Response Unit has elected to deploy the Hapsite as well as the Viking 572 to cover the needs of field responders. The Viking 572 is deployed because it is not limited to gas phase analytes. The use and demand for field GC-MS will continue to grow as these instruments are miniaturized and performance remains at lab-quality. As more of these newer instruments enter the market, the costs will invariably drop to refuel the instrument development cycle.

\section{Acknowledgments}

The author gratefully acknowledges Eric Garff, Jeff Bryson (MMD project), Russ Drew, Eric Qiu, Yuchi Huang, Brian Abraham, and Mark Wilson (Bruker/Viking), Steve DeLuca, Greg Adams, and Pat Dempsey (Inficon), Jean-Luc Truche (Agilent), Todd McCall (FBI Evidence Response Team), and Mark Miller (FBI Forensic Science Research Unit) for their helpful comments and support during these field explorations. The author also thanks O. David Sparkman for helpful comments and assistance in the technical review of this manuscript.

\section{Disclaimer}

This is publication 01-09 of the Laboratory Division of the Federal Bureau of Investigation. Names of commercial manufacturers are provided for identification only and inclusion does not imply endorsement by the FBI.

\section{References}

1. Meuzelaar, H. L. C.; Dworzanski, J. P.; Arnold, N. S.; McClennen, W. H.; Wager, D. J. Field Analytical Chemistry and Technology, 2000, 4, 3-13.

2. Eckenrode, B. A. Field Analytical Chemistry and Technology, 1998, 2, 3-20.

3. Arnold, N. S.; McClennen, W. H.; Meuzelaar, H. L. C. Anal. Chem. 1991, 63, 299-304.

4. Niu, W.; Kuehn, T. Proceedings of the 5th Int. Symposium. on Protection Against Chemical and Biological Warfare Agents, Stockholm, Sweden, June, 1995; pp 43-48.

5 Eckenrode, B.; Niu, W.; Lee, G. Proceedings of the 89th AEWMA, Nashville, TN, May 1996; p A-1326.

6. Niu, W.; Lee, G. Eckenrode, B. Proceedings of the Worldwide Chemical Conference XIV and NBC Operations Symposium, October 1995; pp 1-5.

7. Program Manager for Non-Stockpile Chemical Materiel, Laboratory Quality Control Plan and Procedures for the NonStockpile Chemical Weapons Materiel Program, Monitoring Concept Plan, 1994.

8. Ember, L. R. Chemical Warfare Agent Detectors Probe the Fogs of War, C\&EN, 1994; pp 26-32.

9. Gankin, Y.; Gorshteyn, A.; Smarason, S.; Robbat Jr., A. Anal. Chem. 1998, 70, 1655-1663.

10. Heyl, M. Field Analytical Chemistry and Technology, 1996, 1, 3-12.

11. Mustacich, R. V.; Holland, P. M.; Everson, J. F. Pittsburgh Conference; Atlanta, GA, 1997; \#706.

12. Syage, J. A.; Hanning-Lee, M. A.; Hanold, K. A. Field Analytical Chemistry and Technology, March 2000, 4, 204-215. 